\title{
Fuzzy logic implementation of photo catalytic sensor
}

\begin{abstract}
This paper presents novel method to control output error of the Photo Catalytic Sensor (PCS) using fuzzy interface. Whig and Ahmad in 2014, proposed the spice model of PCS which is a type of ion sensitive FET and very useful to estimate the parameter BOD (Biological Oxygen Demand) which is generally used to estimate quality of water. PCS can also be used to calculate the amount of concentration level of oxygen required to purify the polluted air and sanitization of surface. The purpose to control the output error due to variation in temperature is due to the fact that the fluctuation of $\mathrm{O}_{2}$ influence the threshold voltage, which is internal parameter of FET can manifest itself as a voltage signal at output but as a function of trans conductance gain. Hence, a system controlled by fuzzy logic which controls the output error is proposed. This novel method is very useful because of their simplicity, robustness and successful practical applications. The proposed system is relies on the fast operation of PCS which operates under sub-threshold conditions and reduced computation time. The system is more users friendly and the outcomes of simulation are fairly in agreement with the theoretical estimation.
\end{abstract}

Keywords: photo catalytic sensor (PCS), field effect transistor (FET), fuzzy rules; fuzzy interface
Volume 2 Issue 3 - 2017

\author{
Pawan Whig,' Syed Naseem Ahmad ${ }^{2}$ \\ 'Vivekananda Institute of Professional studies, India \\ ${ }^{2}$ Department of electronics and communication engineering, \\ Jamia Millia Islamia, India
}

Correspondence: Pawan Whig,Vivekananda Institute of Professional studies, India, Email pawanwhig@gmail.com

Received: March 18,2017 | Published: May 16, 2017
Abbreviations: PCS, photo catalytic sensor; FET, field effect transistor, $\mathrm{TiO}_{2}$ titanium oxide; FIS, fuzzy inference engine

\section{Introduction}

Environment plays an important role on the outputs of electronic sensors and systems. Electronic sensors used for very precise measurements perform very well under lab environment. ${ }^{1-2}$ Semiconductor technology is very popular and widely used for sensor development as it provides an advantage of low power, high speed, small size integration and their signal processing capability. More often, CAD tools are used (Sze, 1994) which provide simulation and synthesis of semiconductor sensors. The SPICE has built in models for most semiconductor devices but there is scarcity of appropriate models for semiconductor sensors. Modelling of MOSFETs ${ }^{3,4}$ requires a deep knowledge of the code structure, subroutines and it is strictly linked to a particular version of SPICE. The simplest PCS is $\mathrm{O}_{2}$ sensitive where the sensitive surface is made up of insulator layer like titanium oxide $\left(\mathrm{TiO}_{2}\right)$ exposed to an electrolyte solution. ${ }^{5}$ A p-type semiconductor and $\mathrm{TiO}_{2}$ insulator are placed into aqueous electrolyte solution where the response of PCS to $\mathrm{O}_{2}$ can be explained in terms of photo catalysis. In the submicron level the thickness of gate oxide is very small in dimension. As the dimension of gate oxide decreases this result in tunnelling of charge carriers which significantly increases the leakage current of MOSFETS and results in the decrease of device reliability. ${ }^{6}$ One way to continue reducing dimensions is possible by using materials having much higher permittivity than silicon dioxide.

In these Ion Sensitive types of sensors the output change some times is deterministic in nature and sometimes random. The sensor output recorded is not consistent with the external parameter variations and it changes randomly. ${ }^{7}$ If the sensor output variation is linear or it following some pattern then we can use the linear model which is able to fit to get the desired output but this does not happen most of the time, this will not work as the output behaviour is nonlinear and non-deterministic. ${ }^{8}$ Therefore it is desirable to devise a model which can improve the performance based on mathematical or artificial intelligence techniques. The scope of this paper is to study the behaviour of sensor output signal variations to minimize the output error generated due to temperature variation ${ }^{9}$ In this paper, fuzzy rule base approach has been implemented and it is compared with mathematical curve fitting technique. Fuzzy technique incorporated to solve this kind of engineering problem has produced good results. This approach for modelling random error drift added a new dimension to this kind of engineering problem.

\section{Photo catalysis process}

The process of photo catalysis is a proficient method for degrading organic compounds. Various literatures are available on the different mechanisms and equations involved in the process for gaining a better knowledge..$^{10}$ The semiconductor material consists of two bands which are valence band and conduction band. The energy gap between these two bands is known as band gap given by Eg. The electrons from the valence band jump to conduction band which may be empty when a light of energy higher than band gap energy falls on the semiconductor material. Holes are left behind in the valence band due to excitation of electrons to higher energy band. These holes on reaching the surface of the organic molecule react with water to give $\mathrm{OH}$-radicals for oxidizing the organic pollutants. The dissolved oxygen in the molecular form acts as a scavenger of the photo generated electrons and forms a superoxide radical ion. Titanium oxide has the ability to cause photo-oxidative destruction of the organic pollutants and is non-corrosive in nature due to which it is used as a catalyst in the process. ${ }^{11}$ The oxygen content in any given sample can be determined by observing the change in dissolved oxygen concentration during the process of photo catalysis. In Photo catalysis process a floating gate electrode is used. The sunlight or UV radiations fall on $\mathrm{TiO}_{2}$ which further act as a catalyst to speed up the photo catalysis process. PCS senses the changes in the oxygen concentration and its voltage levels change as an indication. The complete Photo catalysis process is shown in Figure 1.

\section{Photo catalysis sensor}

The SPICE model for PCS is given in Villegas. ${ }^{12}$ It is basically a MOSFET having structural difference in which the gate terminal is kept inside the solution and diffusion and quantum capacitances are added to overcome the effect of Helmholtz and diffusion layer. ${ }^{13-15}$ 
The cross section of PCS is shown in Figure 2. The threshold voltage equation for the PCS model is given as:

$V t h(P C S)=E_{\operatorname{Re} f}-\Psi_{s o l}+X^{s o l}+\frac{-\Phi_{s}}{q}-\frac{Q_{o x}+Q_{s s}+Q_{B}}{C_{o x}}+2 \Phi f$

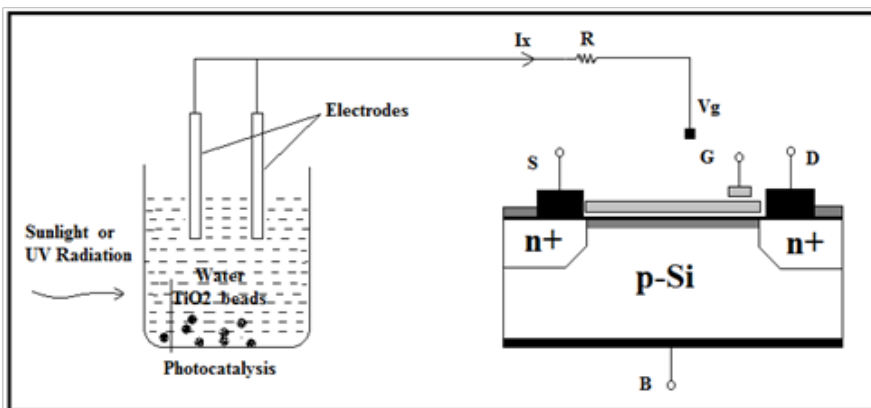

Figure I Photo catalysis process.

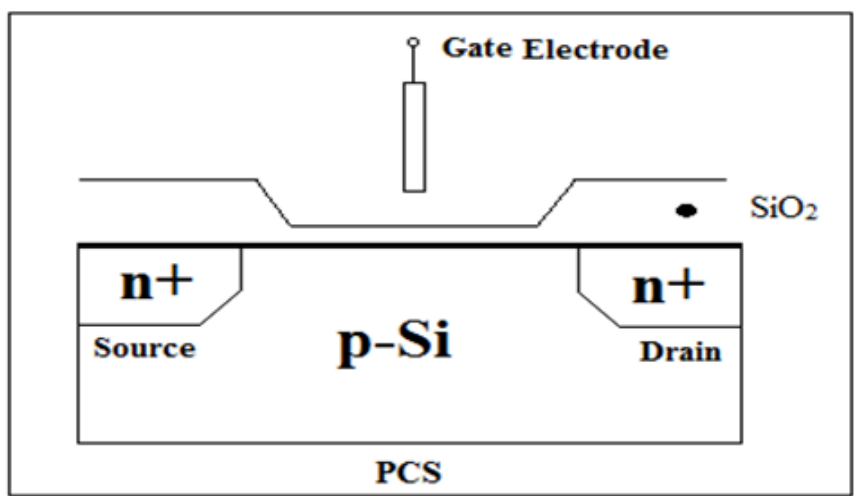

Figure 2 Cross-section of PCS.

$\Psi_{\text {sol }}$ is an input parameter of the equation which is dependent on the concentration of $\mathrm{O}_{2}$ in the solution and surface dipole potential $X^{\text {sol }}$. Here ERef is the constant reference electrode potential. For different concentrations of $\mathrm{O}_{2}$, different $\mathrm{V}-\mathrm{I}$ curves for PCS can be plotted. $\Psi_{\text {sol }}$ is a function of $\mathrm{O}_{2}$ and as the saturation cut-off current Ids increases the value of the oxygen concentration level decreases. The circuit for PCS as given in Peterson ${ }^{14}$ is shown in Figure 3.

Here CM is the resultant of $C_{o x}$ and $C_{q}$ which are oxide and quantum capacitances respectively. The equivalent capacitance $\mathrm{CM}$ is given as:

$$
\frac{1}{C_{M}}=\frac{1}{C_{q}}+\frac{1}{C_{o x}}
$$

The drain current equation in non-saturation mode for PCS is given as:

$$
I_{d s}=C_{o x} \mu \frac{W}{L}\left[\left(V_{g s}-V_{t}\right) V_{d s}-\frac{1}{2} V_{d s}^{2}\right]
$$

Where Cox, Oxide capacitance per unit area; $\mu$, Mobility of electrons in the channel; W, Channel width;

\section{L, Length of the Channel.}

Various Process Parameters including length of channel and channel width are chosen according to the $120 \mathrm{~nm}$ CMOS process model. According to the characteristics of the MOSFET gate to source voltage, Vgs known as reference voltage drain current is allowed to vary with drain to source voltage keeping reference voltage constant. Comparing PCS with MOSFET keeping the concentration of $\mathrm{O}_{2}=1 \mathrm{mg} / \mathrm{l}$ it is found that the curve resembles with the characteristic $\mathrm{V}_{\mathrm{ds}} / \mathrm{I}_{\mathrm{ds}}$ curve of MOSFET keeping $\mathrm{V}_{\mathrm{gs}}$ constant. Now keeping the reference voltage $\mathrm{V}_{\mathrm{gs}}=0$ it is observed that for different concentration levels of $\mathrm{O}_{2}$, different $\mathrm{V}_{\mathrm{ds}} / \mathrm{I}_{\mathrm{ds}}$ curves are obtained as shown in Figure 4. From the above it is observed that as the oxygen concentration level decreases saturation cut off current Ids increases hence it is concluded that PCS can be treated as MOSFET on the basis that the chemical input parameter $\Psi_{\text {sol }}$ is a function of $\mathrm{O}_{2}\left(\Psi_{\text {sol }}=\mathrm{f}(\right.$ Oxygen $\left.)\right)$. For the different values of oxygen content the curves between $\mathrm{I}_{\mathrm{ds}}$ and $\mathrm{V}_{\mathrm{ds}}$ is shown in Figure 4

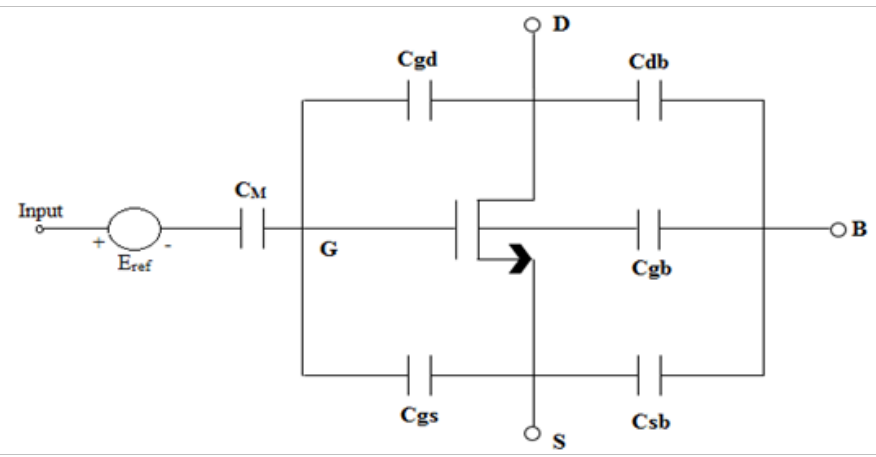

Figure 3 Circuit for PCS.

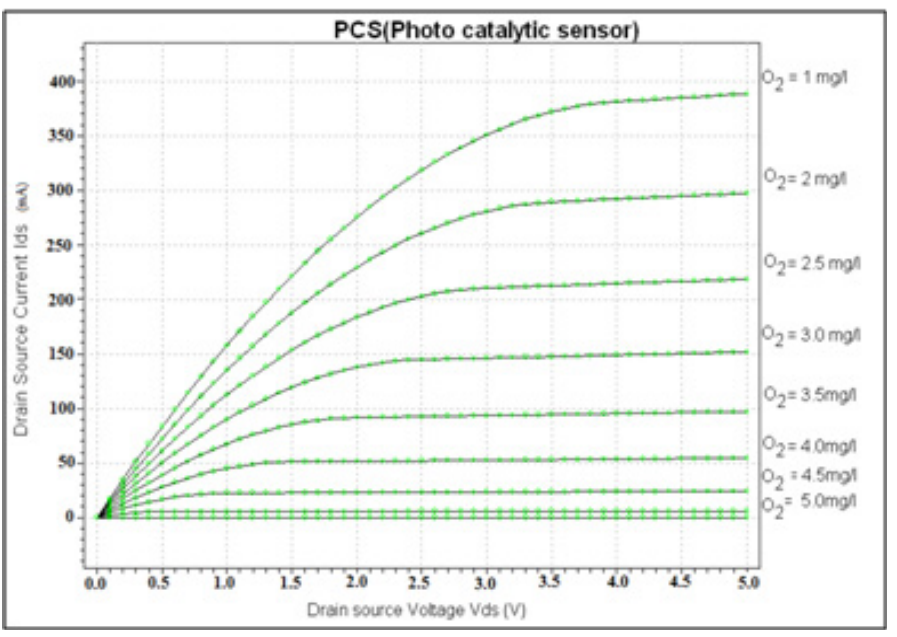

Figure $4 \mathrm{I}_{\mathrm{ds}} \mathrm{V}_{s} \mathrm{~V}_{\mathrm{ds}}$ curve for different value of oxygen content.

\section{Fuzzy implementations}

Fuzzy approach is based upon IF-THEN fuzzy rules. Fuzzy systems can have multiple inputs and single output system. Fuzzy system consist of four major components i.e., fusilier, fuzzy rule base, fuzzy inference engine and at output defuzzifer ${ }^{15}$ Each component has its own role. For example, fuzzy rule base contains the fuzzy IFTHEN rules. Fuzzy inference engine (FIS) using inference method maps the fuzzy sets in the input space to the output space using fuzzy logic. Fusilier and defuzzifier transform the input variables to the fuzzy sets and vice versa respectively. ${ }^{16}$ Fuzzy Rule Base implementation requires first defining the linguistic variables. ${ }^{9,10}$ Based upon our acquired data of photo catalytic sensor, the declaration of fuzzy variables to the inputs and outputs is defined. It is clear from the Table 1 that the temperature varies between 10 to $45^{\circ} \mathrm{C}$. Hence, we have 
defined the temperature range into three categories i.e., Low, Medium and High as given in Table 1. The second input to the fuzzy system is sensor output data in microvolt $(\mu \mathrm{V})$ which act as the input to get the desired output or corrected output. Since the sensor data varies in between 5 to $20 \mu \mathrm{V}$ range as depicted in the Table 1. The third column in the Table 1 shows the fuzzy label for sensor output data range.

Table I Fuzzy Input Labels

\begin{tabular}{lll}
\hline Range & Temperature Range $\left({ }^{\circ} \mathrm{C}\right)$ & Sensor Data Range $(\mu \mathrm{V})$ \\
\hline Low & Low $(10-20)$ & L-SEN $(5-10)$ \\
Medium & Medium $(20-30)$ & M-SEN $(7-17)$ \\
High & High $(30-45)$ & H-SEN $(10-20)$ \\
\hline
\end{tabular}

The output from the fuzzy system will be the desired output or corrected output. Our aim is that sensor output does no change with respect to temperature variation rapidly. It should behave like at room temperature or at Low temperature range. So corrected output should lie in the "L-SEN" range i.e 5 to $10 \mu \mathrm{V}$. We have defined the sensor output range in Low (L-SEN) and Medium (M-SEN) for sensor data range as shown in Table 2. Based upon these fuzzy label declarations, triangular membership function has been chosen and defined. The other membership functions like Gaussian and tepozodial can also be defined. The Input membership function of the Sensor input data is shown below in the Figure 6. Similarly output membership function is defined and it is shown in Figure 7 Based upon the input output membership functions, the fuzzy rules ${ }^{7}$ which will give the desired output from the fuzzy system. Nine possible combinations have been considered at the input side because there are three membership functions for each input. These are listed below which are capable of producing the desired output. Since the two inputs occur simultaneously, so AND operator has been chosen in IF statements.

$\mathrm{R} 1$ : If temperature is low [10-20] AND sensor output is low [5-10] THEN final output is low [5-10]

R2: If temperature is medium [20-30] AND sensor output is low [5-10]

THEN final output is low [5-10] [7-17]

R9: If temperature is high [30-45] AND sensor output is medium

THEN final output is medium [20-30].

Table 2 Fuzzy Output Labels

\begin{tabular}{ll}
\hline Range & Sensor Data Range $(\mu \mathrm{V})$ \\
\hline Low & Low Output(10-20) \\
Medium & Medium Output(20-30)
\end{tabular}

\section{Principle of fuzzy based PCS system}

The performance specifications of the system can be improved by tuning value of various parameters. By self tuning it mean the characteristics of the controller to change or adjust its controlling parameters on-line automatically so as to have the most appropriate values of these parameters, which help system to get desired value. Fuzzy self-tuning works on the basis of control rules, which can be obtained by theoretical and experimental analysis of any system of expert. Thus, fuzzy logic can tune the internal parameters with the help of rule base on-line. This provides better performance than the conventional or simple fuzzy controller.

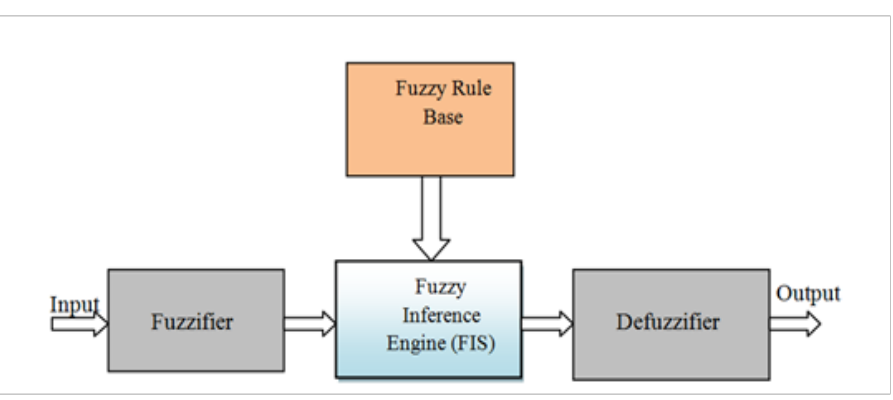

Figure 5 Block diagram of Fuzzy Logic Implementation.

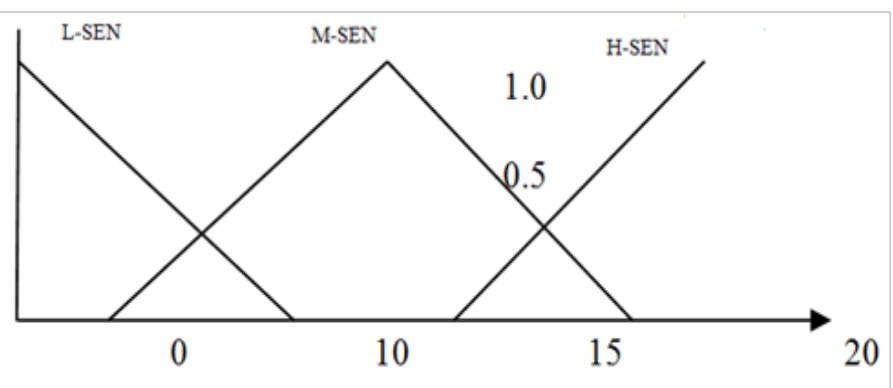

Figure 6 Input Membership function.

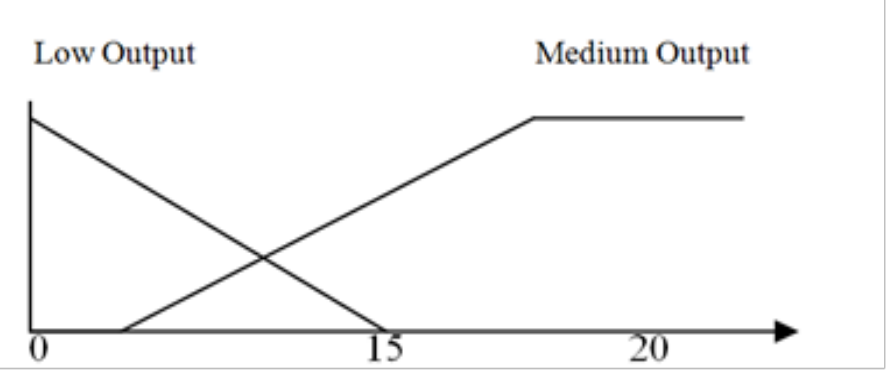

Figure 7 Output membership function.

\section{Design of self-tuning Fuzzy PID Controller}

The self-tuning Fuzzy controller takes "error" (e) and "rate of change of error" (ec) as input to fuzzy logic controller and modify value of three parameters "L-SEN", "M-SEN" and "H-SEN" online as output. Thus we have total of five linguistic variables (e, ec, LSEN, M-SEN, H-SEN). Total of seven fuzzy value NB, NM, NS, $\mathrm{ZO}, \mathrm{PS}, \mathrm{PM}, \mathrm{PB}$ are chosen for each of the linguistic variable. The region "e" and "ec" are between -3 to 3 whereas by doing some interpolation region of L-SEN, M-SEN and H-SEN are kept between 0 and 1. Interpolation tries to keep the value of the variable within specified region. The linguistic rules are important part of FIS. These rules are called the rule base. These rules are created with the help of human knowledge and expertise upon behaviour of the system under different condition. Any number of such rules can be formed to give the controller direction for action.

\section{Results and discussion}

Rules for fuzzy interface system design for different values of $\mathrm{L}-$ SEN, M-SEN and H-SEN are shown in Table 3-5. Thus in a fuzzy 
interface system design there are total of two input as "e" and "ec" and three output as L-SEN , M-SEN and H-SEN as shown in Figure 8 . Hence by these three tables total 49 rules are formed in FIS using MATLAB. Two input three outputs Fuzzy inference system has been designed. Inputs are "error" and "rate of change of error "and outputs are three parameter L-SEN, M-SEN and H-SEN of controller. The transient analysis of Fuzzy system design is shown in Figure 9. The output of fuzzy interface is linear shows that the sensor system is stable and the system is independent from the impact of external parameters. The surface plots corresponding to L, M and H-SEN is plotted with respect to two parameters e and ec as shown in the Figures 10-12. It is observed for the figures that the L-SEN (sensor output range at low temperature) has a little effect of change in e and ec value.

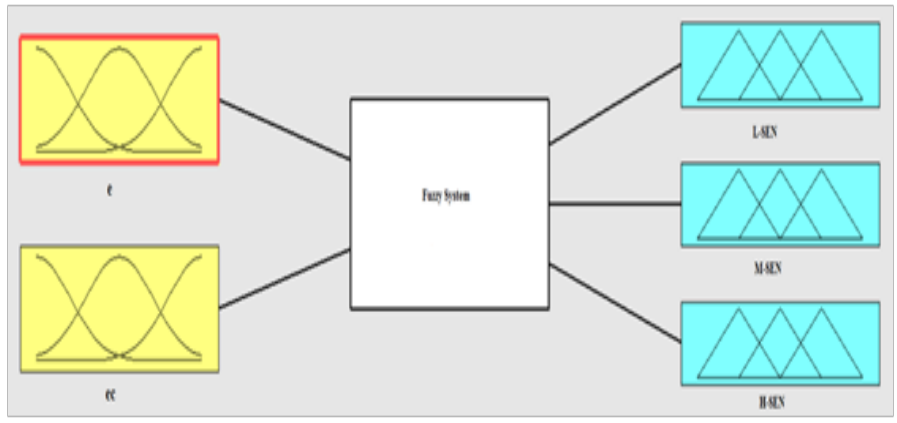

Figure 8 Fuzzy Inference System Design.

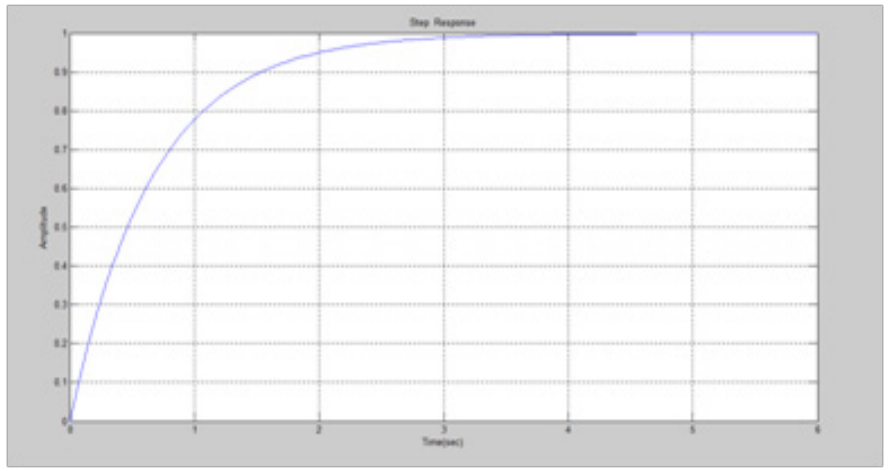

Figure 9 Transient analysis.

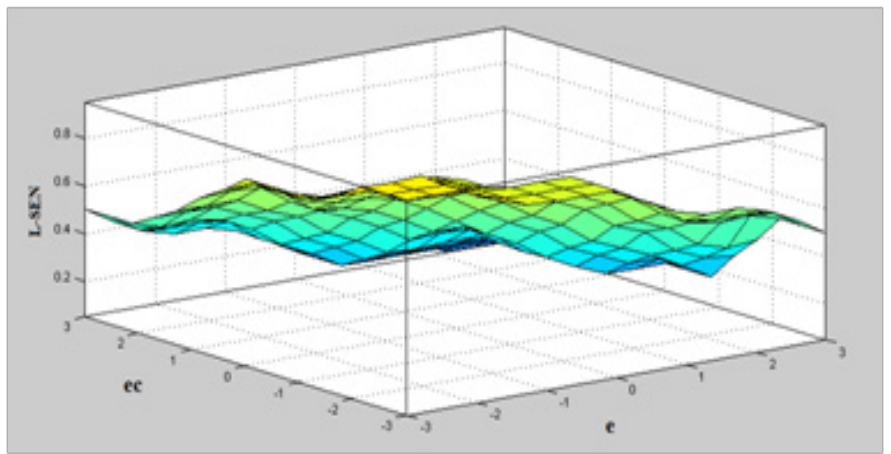

Figure 10 Surface plot corresponding to L-SEN.

\section{Conclusion}

The error control using fuzzy logic interface is presented in this paper. Temperature variation in the environment where these sensors installed degrades the performance due to change in the properties of material. Data has been acquired from sensor under temperature varying environment. The output of the sensor has shown nondeterministic and non-linear behaviour with respect to temperature change. We have developed the fuzzy based technique to model and minimize the output variation. Fuzzy approach has shown better results with Sugeno model with Gaussian membership functions as compared to the Mamdani model. It means that fuzzy approach has ability to produce much better results as compared to other mathematical techniques. In future, Genetic Algorithm can also be implemented to check the results with fuzzy system as a comparison. Also, this study may be extended for further improvements in terms of power and size, besides the wiring and layout characteristics level.

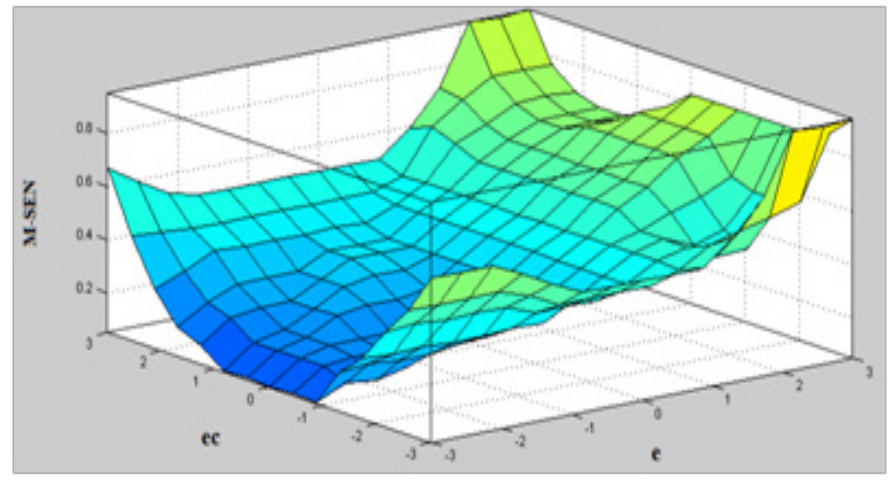

Figure II Surface plot corresponding to M-SEN.

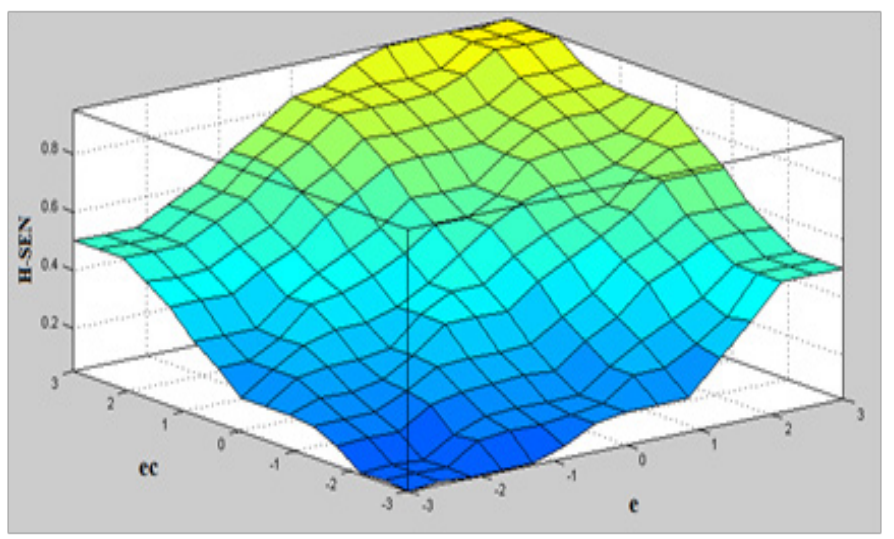

Figure 12 Surface plot corresponding to H-SEN.

Table 3 Rule base for L-SEN

\begin{tabular}{llllllll}
\hline e/ec & NB & NM & NS & ZO & PS & PM & PB \\
\hline NB & PB & PB & PM & PM & PS & ZO & ZO \\
NM & PB & PB & PM & PS & PS & ZO & NS \\
NS & PM & PM & PM & PS & ZO & NS & NS \\
ZO & PM & PM & PS & ZO & NS & NM & NM \\
PS & PS & PS & ZO & NS & NS & NM & NM \\
PM & PS & ZO & NS & NM & NM & NM & NB \\
PB & ZO & ZO & NM & NM & NM & NB & NB \\
\hline & & & & & & &
\end{tabular}


Table 4 Rule base for M-SEN

\begin{tabular}{llllllll}
\hline e/ec & NB & NM & NS & ZO & PS & PM & PB \\
\hline NB & NB & NB & NM & NM & NS & ZO & ZO \\
NM & NB & NB & NM & NS & NS & ZO & ZO \\
NS & NB & NM & NS & NS & ZO & PS & PS \\
ZO & NM & NM & NS & ZO & PS & PM & PM \\
PS & NM & NS & ZO & PS & PS & PM & PB \\
PM & ZO & ZO & PS & PS & PM & PB & PB \\
PB & ZO & ZO & PS & PM & PM & PB & PB
\end{tabular}

Table 5 Rule base for H-SEN

\begin{tabular}{llllllll}
\hline e/ec & NB & NM & NS & ZO & PS & PM & PB \\
\hline NB & PS & NS & NB & NB & NB & NM & PS \\
NM & PS & NS & NB & NM & NM & NS & ZO \\
NS & ZO & NS & NM & NM & NS & NS & ZO \\
ZO & ZO & NS & NS & NS & NS & NS & ZO \\
PS & ZO & ZO & ZO & ZO & ZO & ZO & ZO \\
PM & PB & NS & PS & PS & PS & PS & PB \\
PB & PB & PM & PM & PM & PS & PS & PB \\
\hline
\end{tabular}

\section{Acknowledgments}

None.

\section{Conflict of interest}

Author declares that there is none of the conflicts.

\section{References}

1. Zhang Y, Du C, Zhou Y, et al. Geomagnetic sensor random error modeling and compensation. Information Science and Engineering (ICISE). 2010. p. 6639-6641.

2. Whig P, Ahmad SN. Simulation of linear dynamic macro model of photo catalytic sensor in SPICE. Compel the International Journal of Computation and Mathematics in Electrical and Electronic Engineering. 2013;33(1/2):611-629.

3. Duffy JA. Bonding, energy levels, and bands in inorganic solids. Longman Scientific \& Technica. 1990.
4. Kim YC, Sasaki S, Yano K, et al. Photocatalytic sensor for the determination of chemical oxygen demand using flow injection analysis. Analytica Chimica Acta. 2001;432(2):59-66.

5. Whig P, Ahmad SN. Performance analysis of various readout circuits for monitoring quality of water using analogue integrated circuits. International Journal of Intelligent Systems and Applications. 2012;4(11):91-98.

6. Chow LLW, Yuena MMF, Chanb PCH, et al. Reactive sputtered TiO2 thin film humidity sensor with negative substrate bias. Sensors and Actuators b-Chemical. 2001;76(1-3):310-315.

7. Whig P, Ahmad SN. A novel Pseudo NMOS integrated ISFET device for water quality monitoring. Active and Passive Electronic Components. 2013;10(1):1-6.

8. Whig P, Ahmad SN. Development of economical ASIC for PCS for water quality monitoring. Journal of Circuits, Systems and Computers. 2014;23(6):1-13.

9. Kahng D, Sze SM. A floating gate and its application to memory devices. The Bell System Technical Journal. 1967;46(4):1288-1295.

10. Berg Y, Lande TS, Naess S. Low-voltage floating-gate current mirrors. IEEE International ASIC Conference and Exhibit. 1997. p. 21-24.

11. Lande TS, Wisland DT, Saether T, et al. FLOGIC-floating-gate logic for low-power operation. In Proceedings of the $3^{\text {rd }}$ IEEE International Conference on Electronics, Circuits, and Systems. 1996. p. 1041-1044.

12. Rodriguez-Villegas E. Low Power and Low Voltage Circuit Design with the FGMOS Transistor. IET Circuits, Devices \& Systems Series. 2006.

13. Massobrio G, Antognetti P. Semiconductor Device Modeling with SPICE. 1993. pp. 1-479.

14. Peterson M, Turner J, Nozik A. Mechanistic studies of the photocatalytical behavior of titania particles in photo electrochemical slurry and the relevance to photo detoxification reactions. J Phys Chem. 1991;95(1):221-225.

15. Polycarpou MM, Uber JG, Zhong W, et al. Feedback control of water quality. IEEE Control Systems. 2002;22(3):68-87.

16. Whig P, Ahmad SN.On the performance of ISFET-based device for water quality monitoring. Int J Communications, Network and System Sciences. 2011;4(11):709-719.

17. Whig P, Ahmad SN. DVCC based readout circuitry for water quality monitoring system. International Journal of Computer Applications. 2012;49(22):1-7. 